\title{
Electric Power Demand Forecasting of Jilin Province Based on Secondary Exponential Smoothing Model
}

\author{
Xiao-Dan GUO ${ }^{1}$ and Peng $\mathrm{LI}^{2, \mathrm{~b}, *}$ \\ ${ }^{1}$ Institute of Economic and Technology, State Grid Jilin sheng Electric Power Supply Company, \\ 1427 Pingquan Road, Nanguan District, Changchun, 130062 \\ ${ }^{2}$ Institute of Economic and Technology, State Grid Jilin sheng Electric Power Supply Company, \\ 1427 Pingquan Road, Nanguan District, Changchun, 130062 \\ bm18222148801@163.com \\ ${ }^{*}$ Corresponding author
}

Keywords: Electric power system, Demand forecast, Secondary exponential smoothing method.

\begin{abstract}
Under the background of rapid economic growth, the issue of electric power development has drawn increasing attention. Based on the economic development, energy structure, environment and other factors, this paper analyzes the influence factors of electric power demand. Then this paper develops a prediction model of electric power demand based on the secondary exponential smoothing model. At last, this model is used to predict the electric power demand of Jilin Province. This model is proven to be sufficient accurate with the posterior difference test.
\end{abstract}

\section{Introduction}

With the improvement of the electric power system reform, including electric power generation and trade, and the development of economy, electric power supply has beyond the requirements in recent years. At the same time, the demand of low carbon electric power is increasing with the development of low carbon economy. So, electric power demand forecasting has become more and more important. Also, the development of science and technology provides various theories and methods for electric power demand forecasting, such as the trend analysis approach, regression analysis prediction method, exponential smoothing method, single consumption method, grey model method, foad density method and elastic coefficient method.

Both power generation forecasting and demand forecasting have received more and more attention (Hernandez et al., 2014)[1]. Dong et al. (1997)[2] indicated that Genetic programming was a method did not require the assumption of any functional relationship between dependent and independent variables, and applied it to solve long-term electric power demand forecasting. García-Ascanio and Maté (2010)[3] introduced interval time series methods as a potential tool that was helpful for reduction in power demand forecasting risk. Also, they compared VAR with iMLP and proved it is effective .

Electric power demand can be classified to three kinds: very short term load forecasting from seconds or minutes to several hours, short term load forecasting from hours to weeks, and medium term and long term load forecasting from months to years (Hippert et al., 2001)[4]. Many literatures have paid attention to these fields. For instance, Chang et al. (2011)[5] developed a weighted evolving fuzzy neural network for monthly electric power demand forecasting in Taiwan. Mousavi et al. (2014)[6] presented a new gene expression programming method and used the annual population, stock index, gross domestic product, and total revenue from exporting industrial products to forecast the electric power demand in Thailand.

In long-term electric power demand forecasting, Liu (2011)[7] reviewed some medium and long-term electric power forecasting methods and progressed a new model-dynamic regression model to predict the electric power demand in China. Gul and Qureshi (2013)[8] incorporated economic and demographic activity through Longrange Energy Alternative Planning System (LEAP) to forecast Pakistan's long term electricity consumption for domestic sector. The new 
power has become more and more important in economy. Kim and Kim (2014)[9] considered the new power generation sources and analyzed the long term electric power supply and demand in Korea's Sixth Basic Plan. Also, panel cointegration analysis was applied to the long term and dynamic electric power demand forecasting (Khan and Abbas, 2016)[10].

Although many methods have been presented to long-term electric power demand forecasting and have been proved to be effective, lots of high precision data is required. The long-term power demand forecasting is different from very short-term and short-term power demand forecasting. It requires relative low prediction accuracy and simple and convenient methods. So, this paper applies secondary Exponential Smoothing method to forecast Jilin province's long-term electric power demand.

\section{Secondary Exponential Smoothing Method}

The second exponential smoothing method is a method that makes an exponential smoothing based on an exponential smoothing value. Due to the change of the linear parameters of the time series in different periods, the predicted value coincides well with the original time series value. With secondary exponential smoothing method, the changed trend of the original time series in different time periods is well shown.

First, we calculate the primary and secondary exponential smoothing sequences.

$$
\begin{gathered}
s_{t}^{(1)}=\alpha \mathrm{x}_{t}+(1-\alpha) s_{t-1}{ }^{(1)} \\
s_{t}^{(2)}=\alpha s_{t}^{(1)}+(1-\alpha) s_{t-1}{ }^{(2)}(\mathrm{t}=1,2 \ldots, \mathrm{T})
\end{gathered}
$$

In here, the smoothing factor $\alpha$ can be optimized by analyzing predictive errors. We calculate the intercept and slope of the predicted line with Equations (3) and (4).

$$
\begin{gathered}
\hat{\alpha}_{t}=2 S_{t}^{(1)}-S_{t}^{(2)} \\
\hat{b}_{t}=\alpha\left[S_{t}^{(1)}-S_{t}^{(2)}\right] /(1-\alpha)(t=1,2, \cdots, T)
\end{gathered}
$$

Then, we can make prediction with Equations (5) and (6).

$$
\begin{gathered}
\hat{x}_{t+1}=\hat{\alpha}_{t}+\hat{b}_{t}=(2-\alpha) s_{t}^{(1)} /(1-\alpha)-s_{t}^{(2)} /(1-\alpha), \quad(t=1,2, \cdots, T) \\
\hat{x}_{T+1}=\hat{\alpha}_{T}+\hat{b}_{T} l, \quad(l=1,2, \cdots)
\end{gathered}
$$

Set historical load sequence as: $\left\{x^{(0)}(1), x^{(0)}(2), x^{(0)}(3), \cdots \cdots x^{(0)}(n)\right\}$.We set the forecast value sequence as: $\hat{x}^{(0)}=\left\{\hat{x}^{(0)}(1), \hat{x}^{(0)}(2), \cdots \cdots \hat{x}^{(0)}(n)\right\}$, and define the absolute value of the difference between the actual value $x^{(0)}(\mathrm{k})$ and the calculated value $\hat{x}^{(0)}(k)$ at time $\mathrm{k}$ is the time k's residual value, that is

$$
\in(k)=\left|x^{(0)}(k)-\hat{x}^{(0)}(k)\right|(k=1,2,3,4, \cdots, n) .
$$

The average value of actual value $x^{(0)}(\mathrm{k})(\mathrm{k}=1,2,3 \ldots, \mathrm{N})$ is as $\bar{x}=\frac{1}{n} \sum_{k=1}^{m} x^{(0)}(k)$. The average value of residual value $\in(k)$ is as $\bar{\epsilon}=\frac{1}{m} \sum_{k=1}^{m} \in(k)$. Here, the parameter $\mathrm{m}$ is the number of predicted residuals and $\mathrm{m} \leq \mathrm{n}$, typically. We define historical data variance as $\mathrm{s}_{1}^{2}=\frac{1}{n} \sum_{k=1}^{m}\left(x^{(0)}(k)-\bar{x}\right)^{2}$.The residual variance is $\mathrm{s}_{2}^{2}=\frac{1}{m} \sum_{k=1}^{m}(\in(k)-\bar{\epsilon})^{2}$. We can get the two important data of the difference test, that is, the posterior difference ratio $\mathrm{C}$, small error probability $\mathrm{P}$ with the 
value $p=\left\{|\in(k)-\bar{\epsilon}|<0.6745 s_{1}\right\}$ and $C=s_{2} / s_{1}$. Index $C$ should be kept as small as possible and it increases in $s_{2}$ and decreases in $s_{1}$. That is, the variance of historical data measures the dispersion of historical data. A small $S_{2}$ indicates that the residual variance is small and the residual dispersion is small. The bigger the index $\mathrm{P}$ is, the better the $\mathrm{P}$ is, indicating that the difference between the residual square values is less than the given value.

\section{Jilin Province's Electric Power Demand Forecast}

In this section, we apply the secondary exponential smoothing method to the forecast of electric power demand of Jilin Province based on the annual power consumption data of Jilin Province from 2000-2014. The predict values when $\alpha=0.9, \alpha=0.5$ and $\alpha=0.2$ are shown in Table 1 .

We can compare the actual value with the predict values when $\alpha=0.9, \alpha=0.5$ and $\alpha=0.2$ with Figure 1.

Then, posterior differential calculation is used to comparing the prediction accuracy when $\alpha=0.9$, $\alpha=0.5$ and $\alpha=0.2$.

When $\alpha=0.9, \quad \bar{x}=(291.37+295.08+306.29+\cdots+667.81) / 15=471.07$,

$\bar{\epsilon}=(|291.37-295.08|+|298.05-306.29|+\cdots+|670.10-667.81|) / 14=20.32$, $\mathrm{s}_{1}=\sqrt{\left[(291.37-471.07)^{2}+\cdots+(667.81-471.07)^{2}\right] / 15}=136.68$ and $\mathrm{s}_{2}=\sqrt{\left[(|291.37-295.08|-20.32)^{2}+(|298.05-306.29|-20.32)^{2}+\cdots+(|670.10-667.81|-20.32)^{2}\right] / 14}=16.49$.

According to $C=s_{2} / s_{1}$, we have c=0.1206. Similarly, we have $\mathrm{c}=0.090$ for given $\alpha=0.5$ and $\mathrm{c}=0.2021$ for given $\alpha=0.2$. Also, $\quad p=\left\{|\in(k)-\bar{\epsilon}|<0.6745 s_{1}\right\}=1$ in all cases. Since the prediction value of electric power demand is closest to the actual value when $\alpha=0.5$, we take $\alpha=0.5$. As a result, the electric power demand prediction model of Jilin Province can be expressed as $X_{15+1}=673.1411+23.4739, l=1,2 \ldots$.

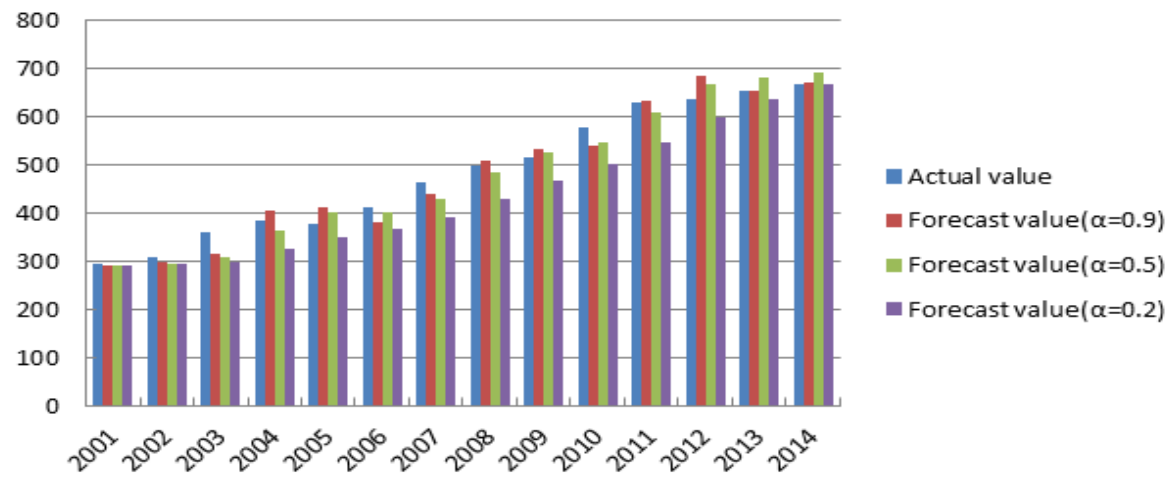

Fig. 1 Comparison between the actual value and prediction value of electric power demand 
Table 1 Comparison of forecast of electric power demand of Jilin Province

\begin{tabular}{ccccc}
\hline \multirow{2}{*}{ Year } & Actual value (million kWh) & \multicolumn{3}{c}{ Forecast value (million kWh) } \\
\cline { 3 - 4 } 2000 & 291.37 & $\alpha=0.9$ & $\alpha=0.5$ & $\alpha=0.2$ \\
2001 & 295.08 & 291.37 & 291.37 & 291.37 \\
2002 & 306.29 & 298.05 & 295.08 & 292.85 \\
2003 & 359.4 & 315.89 & 307.22 & 298.38 \\
2004 & 383.06 & 403.89 & 363.13 & 323.47 \\
2005 & 378.23 & 411.32 & 399.84 & 350.43 \\
2006 & 412.46 & 379.81 & 399.99 & 367.06 \\
2007 & 462.64 & 439.83 & 428.82 & 391.84 \\
2008 & 496.49 & 508.58 & 482.11 & 428.60 \\
2009 & 515.25 & 532.99 & 524.42 & 467.03 \\
2010 & 576.98 & 537.44 & 546.77 & 500.30 \\
2011 & 630.15 & 630.62 & 606.21 & 546.89 \\
2012 & 637 & 683.81 & 666.93 & 599.17 \\
2013 & 653.85 & 653.21 & 679.77 & 636.62 \\
2014 & 667.81 & 670.10 & 689.13 & 667.33 \\
\hline
\end{tabular}

Note: Data Source: Jilin Provincial People's government, http://www.jl.gov.cn/.

Therefore, it can be determined that the accuracy of the model is high, and the model can be used to predict the electricity consumption in Jilin Province. However, the error in the prediction is unavoidable, only to minimize the error and make the result more accurate. The index smoothing coefficient is an uncertain value, the selection is influenced by subjective factors, but generally, the index smoothing coefficient is similar. As shown in Table 1, the predicted value in 2003 is much lower than the actual electricity consumption, and the predicted value is 5.218 billion kwh lower than the actual value, which is perhaps because the implementation of the revitalization strategy of the northeast old industrial in 2003. In addition, through the analysis of the data in Table 1, it is found that the predictive value of other years is controlled within a certain error range, which achieves the expected effect.

In recent years, along with the moderate slowdown of GDP growth, the growth rate of energy and electricity demand in Jilin Province has significantly decreased. According to statistics, as of the end of 2015, the electricity consumption of these industries and residential life is 1.9: 64.4: 17.3: 16.4, the proportion of electricity consumption in the secondary industry is still the largest, followed by the tertiary industry, residents' living and primary industry. In general, the transformation of Jilin Province's electricity demand is closely linked with macroeconomic development, climate change and the development of environmental protection. At present, the power industry in Jilin Province has turned into a medium-low-speed growth. Although the electricity consumption is increasing year by year, the growth rate of electricity consumption has obviously dropped. Economic development in Jilin Province has entered a new normal and structural adjustment is very important to compress and reduce high-energy-consuming industries. Therefore, whether it is a shift from high-speed to medium-high-speed or a growth mode, the adjustment of institutions and the demand for electricity will all drop.

Lastly, we predict the electric power demand if the future which is shown in Table 2. The electric power demand will increase largely in the next fifteen years. It seems reasonable. Firstly, as the industrial structure upgrade in Jilin, the share manufacturing industry in economy will increase. Secondly, the urban population will increase in the future. Lastly, low carbon economy will encourage people and companies to replace coal with electric power. All these will increase electric power consumption. 
Table 2 Forecast value of 2015-2030 years power in Jilin Province

\begin{tabular}{cccc}
\hline Year & Actual value $(\alpha=0.5)($ million $\mathrm{kWh})$ & Year & Actual value $(\alpha=0.5)($ million $\mathrm{kWh})$ \\
\hline 2015 & 696.61 & 2023 & 884.41 \\
2016 & 720.09 & 2024 & 907.88 \\
2017 & 743.56 & 2025 & 931.35 \\
2018 & 767.04 & 2026 & 954.83 \\
2019 & 790.51 & 2027 & 978.30 \\
2020 & 813.98 & 2028 & 1001.78 \\
2021 & 837.46 & 2029 & 1025.25 \\
2022 & 860.93 & 2030 & 1048.72 \\
\hline
\end{tabular}

\section{Conclusion}

The secondary exponential smoothing model is used to predict the electricity consumption in Jilin Province. After the test of the posterior difference, the result of the forecast and the stability of the forecasting result are the best when the smoothing coefficient is 0.5 Close to the actual curve, so this smoothing coefficient is an effective calculation as the basis for future projections. The smoothing coefficient is very important to the forecasting result. Through the test analysis of 2000-2016 electricity consumption in Jilin Province, when the smoothing coefficient $\propto=0.5$ is given, the precision and fitting degree are relatively high. The expectation of this paper is to improve the prediction accuracy of Jilin Province and the saving of resources in Jilin Province, as well as the improvement of investment quality.

\section{Acknowledgement}

This research was financially supported by the Science and Technology Program of STATE GRID Corporation of China (NO. SGJLJY00JJJS1600007).

\section{References}

[1] Hernandez L, Baladron C, Aguiar J M, et al. A Survey on Electric Power Demand Forecasting: Future Trends in Smart Grids, Microgrids and Smart Buildings[J]. IEEE Communications Surveys \& Tutorials, 2014, 16(3):1460-1495.

[2] Dong G L, Lee B W, Chang S H. Genetic programming model for long-term forecasting of electric power demand[J]. Electric Power Systems Research, 1997, 40(1):17-22.

[3] García-Ascanio C, Maté C. Electric power demand forecasting using interval time series: A comparison between VAR and iMLP[J]. Energy Policy, 2010, 38(2):715-725.

[4] Hippert H S, Pedreira C E, Souza R C. Neural Networks for Short-Term Load Forecasting: A Review and Evaluation[J]. IEEE Transactions on Power Systems, 2001, 16(1):44-55.

[5] Chang P C, Fan C Y, Lin J J. Monthly electricity demand forecasting based on a weighted evolving fuzzy neural network approach[J]. International Journal of Electrical Power \& Energy Systems, 2011, 33(1):17-27.

[6] Mousavi S M, Mostafavi E S, Hosseinpour F. Gene expression programming as a basis for new generation of electricity demand prediction models $\hat{\xi}[\mathrm{J}]$. Computers \& Industrial Engineering, 2014, 74:120-128.

[7] Nan L. Based on dynamic regression model medium and long-term demand forecast and analysis of China's electric power[C]// International Conference on Information Science and Engineering. IEEE, 2011:1053-1056. 
[8] Gul M, Qureshi W A. Long term electricity demand forecasting in residential sector of Pakistan[C]// Power and Energy Society General Meeting. IEEE, 2013:1-7.

[9] Kim J, Kim J. Portfolio Analysis on the New Power Generation Sources of the Sixth Basic Plan for Long Term Electricity Demand and Supply[J]. 2014, 23(4):583-615.

[10]Khan M A, Abbas F. The dynamics of electricity demand in Pakistan: A panel cointegration analysis[J]. Renewable \& Sustainable Energy Reviews, 2016, 65(November 2016):1159-1178. 